\title{
PARANORMAL OPERATORS ON BANACH SPACES
}

\author{
N.N. Chourasia and P.B. Ramanujan
}

\author{
In this note we show that a paranormal operator $T$ on a Banach \\ space satisfies Weyl's theorem. This is accomplished by showing \\ that \\ (i) every isolated point of its spectrum is an eigenvalue and \\ the corresponding eigenspace has invariant complement, \\ (ii) for $\alpha \neq 0, \operatorname{Ker}(T-\alpha) \perp \operatorname{Ker}(T-\beta)$ (in the sense of \\ Birkhoff) whenever $\beta \neq \alpha$.
}

\section{Introduction and notations}

$X$ will denote a Banach space and $B(X)$ the Banach algebra of bounded linear operators on $X . T \in B(X)$ will be called paranormal if

$$
\|T x\|^{2} \leq\left\|T^{2} x\right\|\|x\|, \quad \forall x \in X .
$$

We note that an isometry is always paranormal. Also the restriction to an invariant subspace, any scalar multiple and the inverse (if it exists) of a paranormal operator are paranormal. Further, every paranormal operator is normaloid. (By normaloid we mean those operators $T$ for which $\|T\|=r(T)$, the spectral radius of $T$.) For proofs refer to [7], where $T$ is taken to be a paranormal operator in a Hilbert space.

Let $M$ and $N$ be linear subspaces of $X$. Then $M$ is said to be orthogonal to $N$ (in the sense of Birkhoff) and we write $M \perp N$ if $\|x+y\| \geq\|x\|$ for all $x \in M$ and $y \in N$. This is a nonsymmetric relation in a Banach space; but it is equivalent to the usual concept of

Received 24 August 1979. The first author wishes to thank the University Grants Commission, New Delhi, for financial support. 
orthogonality in a Hilbert space ([4], Theorem 2). Let $\sigma(T)$ denote the spectrum of $T$, and $R(T)$ and $N(T)$ its range and null space, respectively. The nullity of $T$ is denoted by $n(T)$ while $\sigma_{p}(T)$ denotes the point spectrum of $T$ and $\zeta(T)$ the complement of $\sigma(T)$.

Let $P_{T}\{\lambda\}$ denote the algebraic eigenprojection associated with $\{\lambda\}$ whenever $\lambda$ is an isolated point of $\sigma(T)$. If $T^{\prime} \in B\left(X^{*}\right)$ is the conjugate of $T \in B(X)$, then

$$
\left(P_{T}\{\lambda\}\right)^{\prime}=P_{T^{\prime}}\{\lambda\}
$$

The corresponding result for a Hilbert space operator $T$ is

$$
\left(P_{T}\{\lambda\}\right)^{*}=P_{T^{*}}\{\bar{\lambda}\},
$$

where * denotes the adjoint and - is complex conjugate.

An operator $T \in B(X)$ is said to be isoloid if every isolated point of $\sigma(T)$ is an eigenvalue of $T$. The numerical range of $T \in B(X)$ is given by

$$
V(B(X), T)=\left\{F(T): F \in B(X)^{*},\|F\|=F(1)=1\right\}
$$

and its numerical radius by

$$
v(T)=\operatorname{Sup}\{|\lambda| \mid \lambda \in V(B(X), T)\} .
$$

If $r(T)$ denotes the spectral radius, then it is known that

$$
r(T) \leq v(T) \leq\|T\|
$$

The operator $T$ is said to satisfy the single valued extension property if $f(\lambda) \equiv 0$ for any $X$-valued analytic function $f$ defined on an open set of the complex plane with $(T-\lambda I) f(\lambda) \equiv 0$.

Denote by $\Pi_{00}(T)$ the set of isolated points $\lambda$ of $\sigma(T)$ for which $0<n(T-\lambda I)<\infty$, and by $\Pi_{O A}(T)$ the set of isolated points $\lambda$ of $\sigma(T)$ for which $R\left(P_{T}\{\lambda\}\right)$ is finite dimensional. Note that $\Pi_{O A}(T) \subseteq \Pi_{0 O}(T)$.

Denote by $W(T)$ Weyl's essential spectrum of $T$ (as defined, for example, in [9], p. 206); let

$$
\sigma_{Z}(T)=W(T) \cup\{\lambda \mid \lambda \text { is a limit point of } \sigma(T)\} .
$$

Note that 


$$
\sigma_{Z}(T)=\sigma(T) \sim \Pi_{\mathrm{O} A}(T)
$$

[6] (here $\sim$ denotes set difference). The operator $T \in B(X)$ is said to satisfy Weyl's theorem if

$$
W(T)=\sigma(T) \sim \Pi_{00}(T)
$$

The famous result of Weyl, that self adjoint operators satisfy (*), has been extended to several classes of operators (see [5] for an account). It is noted in [5] that, among operators related to normal operators, Weyl's theorem does not extend appreciably beyond the seminormal ones. Below we show that for any paranormal operator $T, T$ and $T^{\prime}\left(T\right.$ and $T^{*}$ in the case of a Hilbert space operator $T$ ) satisfy Weyl's theorem, thus extending the theorem beyond the seminormal ones. Also we get a class of operators on Banach space, including isometries, which satisfy Weyl's theorem. In the process we get some results regarding eigenspaces of a paranormal operator.

\section{Main results}

We start with the observation that if $T$ is a paranormal operator on $X$ and $\sigma(T)$ lies on the unit circle then $T$ is an invertible isometry. In fact, $T$ being invertible, both $T$ and $T^{-1}$ are normaloid, being paranormal. Hence $\|T\|=\left\|T^{-1}\right\|=1$ and $\|x\|=\left\|T^{-1} T x\right\| \leq\|I x\| \leq\|x\|$, for all $x \in X$. This shows that $T$ is an invertible isometry.

THEOREM 2.1. If $T$ is a paranormal operator in $B(X)$, then every isolated point of $\sigma(T)$ is a pole of the resolvent $R_{\lambda}(T)$ of order I and the corresponding eigenspace has an invariant complement.

Proof. Suppose $\lambda_{1}$ is an isolated point of $\sigma(T)$. If $\lambda_{1}=0$, consider the paranormal operator $T / R\left(P_{T}\{0\}\right)$. Since $\sigma\left(T / R\left(P_{T}\{0\}\right)=\{0\}\right.$, $T / R\left(P_{T}\{0\}\right)=0$. Thus 0 is a pole of the resolvent $R_{\lambda}(T)$ of order 1 ([11], p. 306). If $\lambda_{1} \neq 0$, consider $T_{1}=\left(1 / \lambda_{1}\right)\left(T / R\left(P_{T}\left\{\lambda_{1}\right\}\right)\right)$. Then $T_{1}$ is a paranormal operator with $\sigma\left(T_{1}\right)=\{1\}$. Thus $T_{1}$ and $T_{1}^{-1}$ both are isometries and $\left\|T_{1}^{n}\right\|=1$ for $n= \pm 1, \pm 2, \pm 3, \ldots$. Also we have 
$T_{1}=I+Q$, where $Q$ is some quasinilpotent operator. It follows from ([3], Section 5, Theorem 3) that $T_{1}=I$. So again $\left(T-\lambda_{1}\right) R\left(P_{T}\left(\lambda_{1}\right)\right)=0$ and $\lambda_{1}$ is a pole of the resolvent $R_{\lambda}(T)$ of order 1 . Thus, using [11], Theorem 5.8A, $R\left(T-\lambda_{1}\right)$ is closed and $X=R\left(T-\lambda_{1}\right) \oplus N\left(T-\lambda_{1}\right)$. This completes the proof of the theorem.

COROLLARY 2.2. If $T \in B(X)$ is paranormal, then $T$ is isoloid. COROLLARY 2.3. If $T \in B(X)$ is an isometry, then every isolated point of $\sigma(T)$ is an eigenvalue of $T$ and the corresponding eigenspace has an invariant complement.

REMARK 2.4. The above result was proved in [8] for invertible isometries on a normed linear space.

COROLLARY 2.5. If $T \in B(X)$ is paranormal, then $\Pi_{0 O}(T)=\Pi_{O A}(T)$ and $\Pi_{O O}\left(T^{\prime}\right)=\Pi_{O A}\left(T^{\prime}\right)$. In the case of a Hilbert space paranormal operator $T$ we have

$$
\Pi_{O O}\left(T^{*}\right)=\Pi_{O A}\left(T^{*}\right)
$$

Proof. If $\lambda_{1}$ is an isolated point of $\sigma(T)$, then $\lambda_{1}$ is a pole of the resolvent $R_{\lambda}(T)$ of order 1 . Thus $\left(T-\lambda_{1}\right) P_{T}\left\{\lambda_{1}\right\}=0$, so that $R\left(P_{T}\left\{\lambda_{1}\right\}\right) \subseteq N\left(T-\lambda_{1}\right)$, which implies that $\Pi_{0 O}(T) \subseteq \Pi_{O A}(T)$. If $\lambda_{1}$ is an isolated point of $\sigma\left(T^{\prime}\right)$, then $\lambda_{1}$ is a pole of the resolvent $R_{\lambda}(T)$ of order 1 . Thus $\left(T-\lambda_{1}\right) P_{T}\left\{\lambda_{1}\right\}=0$. Taking conjugates, $\left(P_{T}\left\{\lambda_{1}\right\}\right)^{\prime}\left(T-\lambda_{1}\right)^{\prime}=0$. Now $\left(P_{T}\left\{\lambda_{1}\right\}\right)^{\prime}=P_{T^{\prime}}\left\{\lambda_{1}\right\}$ and $P_{T},\left\{\lambda_{1}\right\}$ commutes with $T^{\prime}$. Hence $\left(T^{\prime}-\lambda_{1}\right) P_{T^{\prime}}\left(\lambda_{1}\right)=0$, so that $\Pi_{0 O}\left(T^{\prime}\right) \subseteq \Pi_{O A}\left(T^{\prime}\right)$. The corresponding result for $T^{*}$ can be similarly proved by taking adjoints.

THEOREM 2.6. If $T$ is a paranormal operator, then $N(T-\alpha) \perp N(T-\beta)$ for distinct complex numbers $\alpha$ and $B$ provided $\alpha \neq 0$.

Proof. Suppose $|\alpha| \geq|\beta|$. Let $T x=\alpha x$ and $T y=B y$. We will show that $\|x\| \leq\|x+y\|$. Let $M$ be the subspace generated by $x$ and $y$ and $T_{1}=T / M$. Then $\sigma\left(T_{1}\right)=(\alpha, B)$ and $T_{1}$ being normaloid, $\left\|T_{1}\right\|=r\left(T_{1}\right)=|\alpha|$. Hence, $v\left(T_{1}\right)=|\alpha|$. Thus, $\alpha \in b d\left(V\left(B(M), T_{1}\right)\right)$. 
Now, by [10], Proposition 1, N( $\left.T_{1}-\alpha\right) \perp R\left(T_{1}-\alpha\right)$. As $\alpha$ and $B$ are poles of the resolvent $R_{\lambda}\left(T_{1}\right)$ of order $l$,

$$
R\left(T_{1}-\alpha\right)=R\left(I-P_{T_{1}}\{\alpha\}\right)=R\left(P_{T_{1}}\{\beta\}\right)=N\left(T_{1}-\beta\right)
$$

([11], Theorem $5.8(\mathrm{~A}))$. Now, $x \in N\left(T_{1}-\alpha\right)$ and $y \in N\left(T_{1}-\beta\right)$. Hence $\|x+y\| \geq\|x\|$. If $|\alpha|<|\beta|, \beta \neq 0$ as $\alpha \neq 0$. So $T_{1}$ is invertible and $\sigma\left(T_{1}^{-1}\right)=\left(\alpha^{-1}, B^{-1}\right)$ and $\left|\alpha^{-1}\right|>\left|B^{-1}\right|$. Being paranormal, $T_{1}^{-1}$ is also normaloid. As in the first case we see that $N\left(T_{1}^{-1}-\alpha^{-1}\right) \perp N\left(T_{I}^{-1}-\beta^{-1}\right)$. As $x \in N\left(T_{1}^{-1}-\alpha^{-1}\right)$ and $y \in N\left(T_{1}^{-1}-\beta^{-1}\right)$, the proof is complete.

$$
\text { QUESTION 2.7. Is } N(T) \perp N(T-\alpha) \text { ? }
$$

REMARK 2.8. It is shown in [8], Corollary 3, that, for an isometry $T$ on a normal linear space, $N(T-\alpha) \perp N(T-\beta)$ for distinct scalars $\alpha$ and $\beta$. This can be shown by completing the normal linear space, extending the isometry to the completed space and using Theorem 2.6.

COROLLARY 2.9. If $T$ is a paranormal operator on a Hilbert space, then $N(T-\alpha) \perp N(T-\beta)$ for all distinct complex numbers $\alpha$ and $\beta$.

COROLLARY 2.10. If $X$ is a separable Banach space and $T \in B(X)$ is paranormal, then $T$ has single valued extension property.

Proof. We will show that $\sigma_{p}(T)$ is countable. Then, by [1], p. 22, $T$ has single valued extension property. If $\sigma_{p}(T)$ is not countable, we would have an uncountable set of unit vectors such that $\left\|x_{i}-x_{j}\right\| \geq 1$. Since the space is separable, this is not possible.

In [8] it is pointed out that for an arbitrary Banach space, it is not known whether an eigenspace of an isometry has an invariant complement. In [2], it is shown that the answer is affirmative for an orthogonally complemented isometry on a smooth reflexive Banach space. We improve this result by using results in [10].

COROLLARY 2.11. If $T$ is an isometry on a reflexive Bonach space, then every eigenspace of $T$ has an invariant complement. 
Proof. If $T$ is an isometry and $\lambda$ is an eigenvalue of $T$, then $|\lambda|=I$ and $\|T\|=r(T)=v(T)=1$. Thus

$$
\lambda \in b d(V(B(X), T)) .
$$

As $X$ is reflexive, by Remark 4 of [10], $X=N(T-\lambda) \oplus R(T-\lambda)$. This completes the proof.

THEOREM 2.12. If $T$ is a paranormal operator on a Banach space, then $T$ and $T^{\prime}\left(T\right.$ and $T^{*}$ in the case of a Hilbert space operator $T$ ) both satisfy Weyl's theorem.

Proof. In view of Theorem 3.3 of [6] and Corollary 2.5, it is enough to show that $W(T)=\sigma_{Z}(T)$. This would prove the result for $T$. Since $W(T)=W\left(T^{\prime}\right)$ and $\sigma_{\ell}(T)=\sigma_{\ell}\left(T^{\prime}\right)$, the result would follow for $T^{\prime}$ and the relations $\overline{W(T)}=W\left(T^{*}\right)$ and $\overline{\sigma_{\eta}(T)}=\sigma_{\eta^{(}}\left(T^{*}\right)$ would yield the result for $T^{*}$. We would show that every $\lambda$ in $\sigma(T) \sim W(T)$ satisfies the eigenspace gap condition of [5]. Then by Theorem 1 (4i) of [5], we get $\sigma(T) \sim W(T) \subseteq \Pi_{00}(T)$. From this it follows easily that $\sigma_{\eta}(T)=W(T)$. Note that $\sigma(T) \sim W(T)=\Pi_{O A}(T) \cup\left\{\sigma_{Z}(T) \sim W(T)\right\}$. If $\lambda \in \pi_{O A}(T)$, then we can find a sequence $\left\{\lambda_{n}\right\}$ such that $\lambda_{n} \epsilon \zeta(T)$ for all $n$ as $\lambda$ is an isolated point of $\sigma(T)$. Thus the gap condition is satisfied here. Given $\lambda$ in $\sigma_{2}(T) \sim W(T)$, we can find a sequence $\left\{\lambda_{n}\right\}$ of nonzero eigenvalues of $T$ converging to $\lambda$. Now $N\left(T-\lambda_{n}\right) \perp N(T-\lambda)$ for all $n$ by Theorem 2.6. So $d\left(x_{\lambda_{n}}, N(T-\lambda)\right) \geq 1$ for all $x_{\lambda_{n}}$ in $N\left(T-\lambda_{n}\right)$ such that $\left\|x_{\lambda_{n}}\right\|=1$. Thus

$$
\begin{aligned}
\delta\left(\lambda_{n}, \lambda\right) & \equiv \operatorname{Sup}\left\{d\left(x_{\lambda_{n}}, N(T-\lambda) \mid x_{\lambda_{n}} \in N\left(T-\lambda_{n}\right) \text { and }\left\|x_{\lambda_{n}}\right\|=1\right\}\right. \\
& \geq 1 .
\end{aligned}
$$

This will be true for all $n$. Hence

$$
\left|\lambda_{n}-\lambda\right| / \delta\left(\lambda_{n}, \lambda\right) \rightarrow 0 \text { as } n \rightarrow \infty
$$

This completes the proof.

PROPOSITION 2.13. If $T$ is a paranormal operator with finite spectrom, then there exists a basis of $X$ consisting of eigenvectors of 
$T$; that is, $X$ is a direct sum of the eigenspaces of $T$.

Proof. Using Theorem 5.7 (A) of [11], we see that

$$
X=\oplus \sum_{i=1}^{n} R\left(P_{T}\left(\left\{\lambda_{i}\right\}\right)\right)
$$

where $\sigma(T)=\left\{\lambda_{1}, \ldots, \lambda_{n}\right\}$, since $\sigma(T)=\bigcup_{i=1}^{n}\left\{\lambda_{i}\right\}$ and each $\left\{\lambda_{i}\right\}$ is an open closed subset of $\sigma(T)$, with $\sigma(T)$ being finite. But

$$
R\left(P_{T}\left\{\lambda_{i}\right\}\right)=N\left(T-\lambda_{i}\right) \quad(i=1, \ldots, n)
$$

since each $\lambda_{i}$ is a pole of resolvent of order 1 by Theorem 2.1. Thus

$$
X=\oplus \sum_{i=1}^{n} N\left(T-\lambda_{i}\right)
$$

This completes the proof.

COROLLARY 2.14. If $T$ is a paranomal operator on a Hilbert space with finite spectrom, then $T$ is normal.

Proof. $X=\oplus \sum_{i=1}^{n} N\left(T-\lambda_{i}\right)$ and $N\left(T-\lambda_{i}\right) \perp N\left(T-\lambda_{j}\right)$ for $i \neq j$ give the result.

COROLLARY 2.15 ([8], Corollary 4). If $T$ is an isometry on a finite dimensional normed linear space $X$, then $X$ has a basis consisting of eigenvectors of $T$.

Proof. This follows directly from Proposition 2.12 because a finite dimensional normed linear space is a Banach space.

\section{References}

[1] Ion Colojoară and Ciprian Foias, Theory of generalised spectral operators (Gordon and Breach, New York, London, Paris, 1968).

[2] G.D. Faulkner and J.E. Huneycutt, Jr, "Orthogonal decomposition of isometries in a Banach space", Proc. Amer. Math. Soc. 69 (1978), $125-128$. 
[3] S.R. Foguel, "The relations between a spectral operator and its scalar part", Pacific J. Math. 8 (1958), 51-65.

[4] J.R. Giles, "Classes of semi-inner-product spaces", Trans. Amer. Math. Soc. 129 (1967), 436-446.

[5] Karl Gustafson, "Necessary and sufficient conditions for Weyl's theorem", Michigan Math. J. 19 (1972), 71-8I.

[6] Karl Gustafson, "On algebraic multiplicity", Indiana Univ. Math. J. 25 (1976), 769-781.

[7] Vasile Istrăłescu, Teishirô Saitô and Takashi Yoshino, "On a class of operators", Tôhoku Math. J. 18 (1966), 410-413.

[8] D. Koehler and Peter Rosenthal, "On isometries of normed linear spaces", Studia Math. 36 (1970), 213-216.

[9] Martin Schechter, "On the essential spectrum of an arbitrary operator. I", J. Math. Anal. Appl. 13 (1966), 205-215.

[10] Allan M. Sinclair, "Eigenvalues in the boundary of the numerical range", Pacific J. Math. 35 (1970), 231-234.

[11] Angus E. Taylor, Introduction to functional analysis (John Wiley \& Sons, New York, London, Sydney, 1958).

Department of Mathematics,

Sardar Patel University,

Vallabh Vidyanagar - 388120 ,

Gujarat,

India;

Department of Mathematics,

Saurashtra University,

Rajkot - 360005,

Gujarat,

India. 